\title{
Do Silicon Ultrasonic Transducers Dream of Becoming Mobile Tactile Display?
}

\author{
Katsuya Shibata ${ }^{1}$, and Naoki Kondo ${ }^{1}$
}

\begin{abstract}
In today's research for the better human-computer interfaces, tactile sensation is one of the most technologically immature, yet the most intensely studied topics. Ultrasonic sound waves as the sensation generator are commonly adopted. Conventional ultrasonic sound source devices employ piezoelectric transducers and thus suffer from the cost and integration issues. In this work we examined theoretically the possibility of exploiting the capacitive micromachined ultrasonic transducers (CMUTs) as a tactile display device that generates two-dimensional pressure distribution above their surface via near-field acoustic radiation pressure effect. We found that with slight modification to existing CMUT geometry, $300 \mathrm{~Pa}$ necessary for invoking tactile sensation is achieved with the spatial resolution of $3.8 \mathrm{~mm}$ close to the average two-point discrimination threshold of $3 \mathrm{~mm}$. The possibility to go further below the $3 \mathrm{~mm}$ threshold by halving the CMUT radius is also pointed out.
\end{abstract}

Keywords - tactile display, CMUT, near-field acoustic radiation pressure.

\section{INTRODUCTION}

A Mong all the senses of human beings, vision and auditory sense are the ones which already enjoy satisfactory sensation methods today. For the others including tactile sense, such methods are still on the way of development. Methods to provide desired tactile sensation had been searched for well over 2 decades mainly through the use of ultrasonic sound waves generated by piezoelectric transducers.

Representative examples among many are such as a single oscillator-based tactile display combined with force display [3] and a scanning-type display with phased transducer arrays [4]. The former uses near-field acoustic radiation pressure for sensation which is energy-efficient and easy to generate. However, it basically cannot provide lateral pressure distribution. The latter allows large positional flexibility of the target, but the fabrication of large piezoelectric array is technologically and economically challenging, thus it is hard to foresee they get implemented in consumer electronics.

We here propose to employ the capacitive micromachined ultrasonic transducers as ultrasonic sound source devices and use their near-field acoustic radiation pressure for tactile sensation.

Katsuya Shibata, and Naoki Kondo, are with Graduate School of Science and Engineering, Teikyo University, Utsunomiya, Japan

\section{A. Capacitive Micromachined Ultrasonic Transducers}

Ultrasonic Transducers were made from piezoelectric materials. Since the material is not for general electronics, their integration into large arrays tends to be technologically and economically demanding. Silicon-based capacitive micromachined ultrasonic transducers whose development became fully in progress in the 1990s [9] are the new types of ultrasonic transducers each of which are composed of a silicon thin plate deflected by the electric force between the plate and the bottom electrode. They are fabricated by the very same process technologies with the silicon integrated circuits, which leads to the fact that they are easy to integrate among themselves as arrays and with the accompanying electronics, thus potentially suited for future consumer-level electronic products.

\section{B. Near-Field Acoustic Radiation Pressure}

Near-field acoustic radiation pressure is a DC-like pressure generated near the sound source and is a second-order nonlinear effect. Far-field acoustic radiation pressure has been known from early days but little attention other than scientific interest was paid since it was so weak that applications are limited to the manipulation of the ultra lightweight objects such as liquid droplets.

However, in the mid-1990s Ueha et al. found experimetarily that large objects of kilogram-order weight can be lifted by the acoustic radiation pressure in the proximity of oscillating surface [1]. Today several applications such as contactless wafer transfer in clean rooms is expected and the phenomena is intensely studied.

Recently direct determination of near-field radiation pressure distribution was made possible for the first time by our group [2].

\section{THEORIES FOR ANALYSIS}

Here we introduce two theories that each describes the behavior of CMUT deflection and near-field acoustic radiation pressure.

\section{A. CMUT Analytical Deflection Theory}

Wygant et al. gave analytical theory of CMUT deflection [7]. If there are atmospheric pressure $P_{a}$ and electric force $F_{e}(t)$ acting on the circular plate of radius $r_{0}$, the total pressure $P_{0}$ is, 


$$
P_{0}=P_{a}+\frac{F_{e}(t)}{\pi r_{0}^{2}}
$$

Then the plate deflection profile $w(r)$ is [8],

$$
w(r)=\frac{P_{0} r_{0}{ }^{4}}{64 D}\left(1-\frac{r^{2}}{r_{0}{ }^{2}}\right)^{2}
$$

with $\mathrm{D}$ being flexural rigidity determined as,

$$
D=\frac{E t^{3}}{12\left(1-v^{2}\right)}
$$

where $E, v$ are the plate material's Young's modulus and Poisson ratio and $\mathrm{t}$ is plate thickness, respectively.

Note that the natural resonance frequency $\omega_{0}$,

$$
\omega_{0}=\frac{10.22}{r_{0}^{2} \sqrt{\rho t / D}}
$$

determines the system's resonance to external AC field.

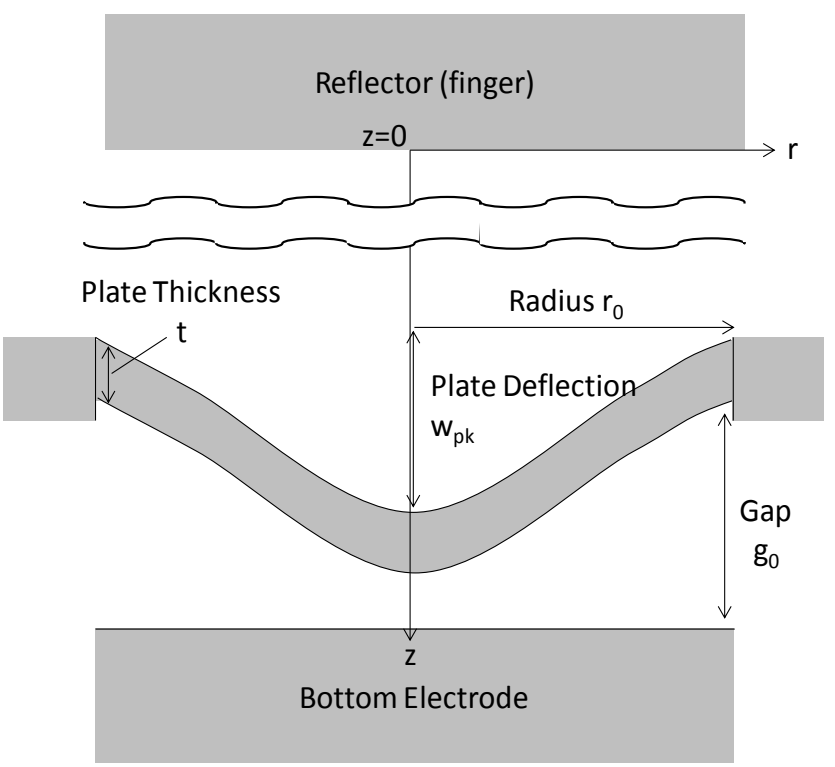

Fig. 1: CMUT model

\section{B. Near-Field Acoustic Radiation Pressure Theory}

$\mathrm{Li}$ et al. gave a refined theory for near-field acoustic radiation pressure that includes both the effects of surface profile and gas inertia [6]. Firstly the momentum equation of Newtonian fluids with inertia is described in axisymmetric coordinates as in Eq. (6):

$$
\rho\left(\frac{\partial v_{r}}{\partial t}+v_{r} \frac{\partial v_{r}}{\partial r}+v_{z} \frac{\partial v_{r}}{\partial z}\right)=-\frac{\partial p}{\partial r}+\frac{\partial \tau_{r z}}{\partial z}
$$

where $\tau_{r z}=\mu \partial v_{r} / \partial z$ is shear stress, $p, \rho$ and $\mu$ are gas pressure, density and viscosity, respectively. Since the air layer is thin, $\mathrm{z}$-dependence of pressure is assumed to be zero, $\frac{\partial p}{\partial z}=0$. The continuity equation is

$$
\frac{1}{r} \frac{\partial}{\partial r}\left(r v_{r}\right)+\frac{\partial v_{z}}{\partial z}=0
$$

If the reflector is flat at $\mathrm{z}=0$ and the vibrator height profile is given by height function $h(r, t)$, then the boundary conditions are,

$$
\begin{aligned}
& v_{r}(r, 0, t)=0 \\
& v_{r}(r, h, t)=0 \\
& v_{z}(r, 0, t)=0 \\
& v_{z}(r, h, t)=d h / d t .
\end{aligned}
$$

Assuming the inertia force can be approximated by its average across its thickness, Eq. (5) becomes

$\frac{\rho}{h} \int_{0}^{h}\left(\frac{\partial v_{r}}{\partial t}+v_{r} \frac{\partial v_{r}}{\partial r}+v_{z} \frac{\partial v_{r}}{\partial z}\right) d z=-\frac{\partial p}{\partial r}+\mu \frac{\partial^{2} v_{r}}{\partial z^{2}}$.

$v_{r}$ has analytical solution of the form Eq. (9),

$$
v_{r}=\frac{3 r}{h^{3}} z(z-h) \frac{d h}{d t}
$$

which leads to the integrable form of $\partial p / \partial r$, namely,

$$
\frac{\partial p}{\partial r}=\frac{6 \mu r}{h^{3}} \frac{d h}{d t}-\frac{9}{10} \frac{r p}{h^{2}}\left(\frac{d h}{d t}\right)^{2}+\frac{r p}{2 h} \frac{d^{2} h}{d t^{2}}
$$

Integrating Eq. (11) in $r$ with the boundary condition,

$$
p\left(r_{0}, t\right)=p_{a}
$$

or

$$
\Delta p\left(r_{0}, t\right)=0
$$

where $\Delta p=p-p_{a}$ gives pressure $p$. Finally the near-field acoustic radiation force $F$ is simply,

$$
F=\int_{0}^{r_{0}} 2 \pi r\left[\frac{1}{T} \int_{0}^{T} \Delta p(r, t) d t\right] d r .
$$

\section{RESULTS AND DISCUSSIONS}

As for the device parameters for our calculation we basically employ airborne CMUT design parameters by Yilmaz [5]. They are listed in Table I. Note however, the plate thickness is altered ranging from realistic $80 \mu \mathrm{m}$ to its half value $40 \mu \mathrm{m}$. Plate radius is $1.9 \mathrm{~mm}$ so that the distance between neighboring CMUTs are $3.8 \mathrm{~mm}$ at minimum which is close to the average $3 \mathrm{~mm}$ two-point discrimination threshold of human tactile sense [10].

TABLE I: EMPLOYED DIMENSIONS OF CMUT MODEL

\begin{tabular}{cll}
\hline \hline Parameter Name & Description & Value \\
\hline$r_{0}$ & Plate radius (m) & $1.9 \times 10^{-3}$ \\
$t$ & Plate thickness (m) & $40-80 \times 10^{-6}$ \\
$P_{e} / P_{a}$ & $\begin{array}{l}\text { Normalized exerted } \\
\text { pressure }\end{array}$ & 0.64 \\
\hline \hline
\end{tabular}

In order for CMUTs to transmit ultrasonic sound waves, there must be space for the air between CMUTs and finger surface. According to the experimental result shown in Fig. 3 
of [3] the friction between finger and flat oscillator surface disappears (which implies that the finger and the oscillator surface becomes contactless) when the displacement amplitude is $5 \mu \mathrm{m}$. We calculated the mean distance between the finger and the oscillator plate from the high squeeze number theory in [1] as $22 \mu \mathrm{m}$ in this situation. This leads to the minimum contactless distance of $17 \mu \mathrm{m}$ subtracting displacement amplitude $5 \mu \mathrm{m}$ from the calculated mean distance $22 \mu \mathrm{m}$. We employed this value for the initial distance between the CMUT plate and the finger surface. In reality we need to put in a spacer layer of this thickness between CMUTs and the finger to ensure the desired discrepancy. Exertion frequency is set to the mechanical resonance frequency determined from Eq. (4).

Calculated squeeze numbers taking their values between 7 and 14 in this setup assures the validity of Li's theory of near-field acoustic radiation pressure which matches with the experimental results in low squeeze number regime. Now that we became ready with necessary parameters, we have calculated the near-field acoustic pressure distribution changing the CMUT plate thickness from $80 \mu \mathrm{m}$ to $40 \mu \mathrm{m}$. The result is shown in Fig. 2.

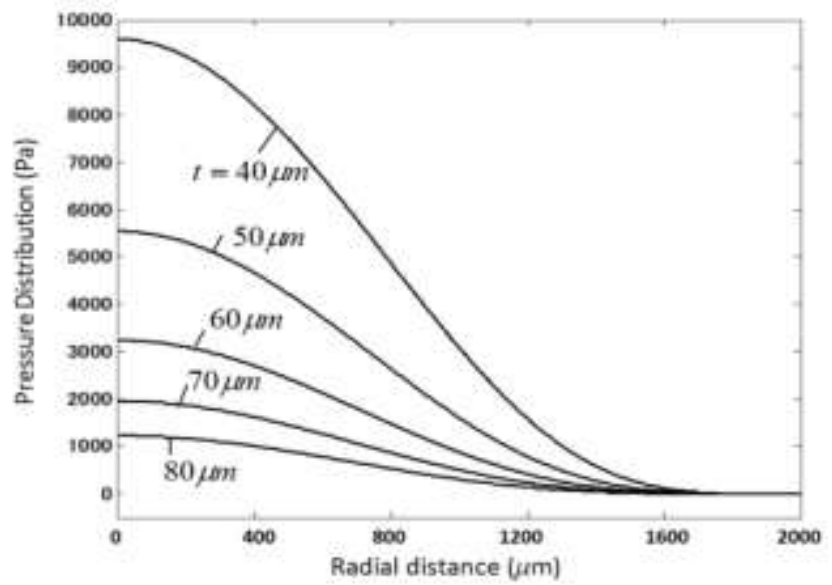

Fig. 2: Radial distribution of near-field acoustic radiation pressure

The form of the radial pressure distribution peaks at the center of the CMUT plate and gradually lowers to zero at the plate edge which differs significantly from the near-flat distribution seen in the high squeeze number regime. Pressure at the center is $1.2 \mathrm{kPa}$ even when the plate is thickest $80 \mu \mathrm{m}$ and rises to $9.5 \mathrm{kPa}$ when the thickness is halved.

Averaged pressure over circular plate area and peak displacement of the plate center against plate thickness is shown in Fig. 3 and 4 respectively. We can see that experimentally realized CMUT in [5] is already capable of generating $200 \mathrm{~Pa}$. To achieve $300 \mathrm{~Pa}$ which is enough for tactile sensation [4], we need to thin down the plate to $73 \mu \mathrm{m}$. Peak displacement is $4 \mu \mathrm{m}$ in this situation. If we go further to demand $1 \mathrm{kPa}$, it can be achieved by thinning the plate down to $50 \mu \mathrm{m}$. However, if we see Fig. 4 the peak displacement becomes $13 \mu \mathrm{m}$. Since this requires serious geometrial reconsideration of the device and evaluation on reliability, it should be thought as quite challenging.

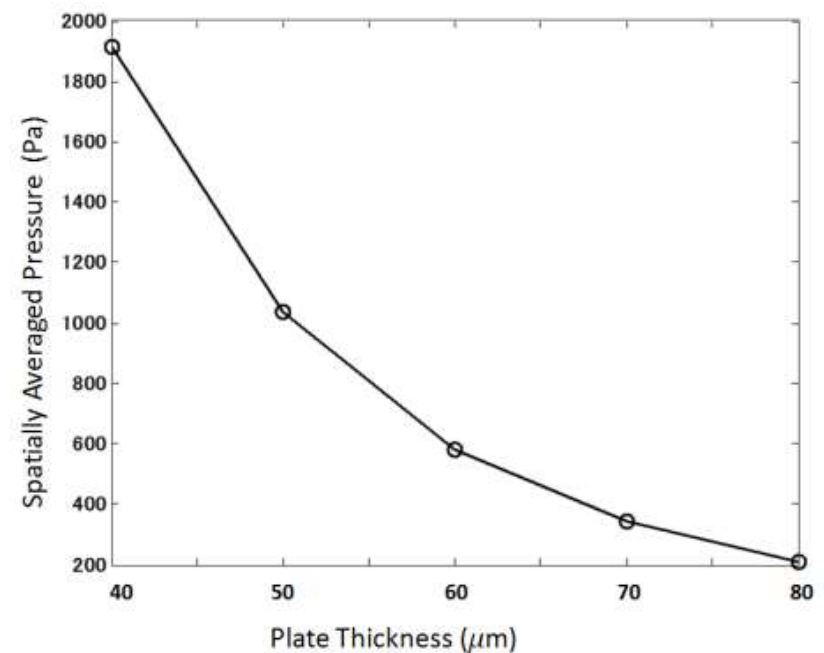

Fig. 3: Spatially averaged near-field acoustic radiation pressure

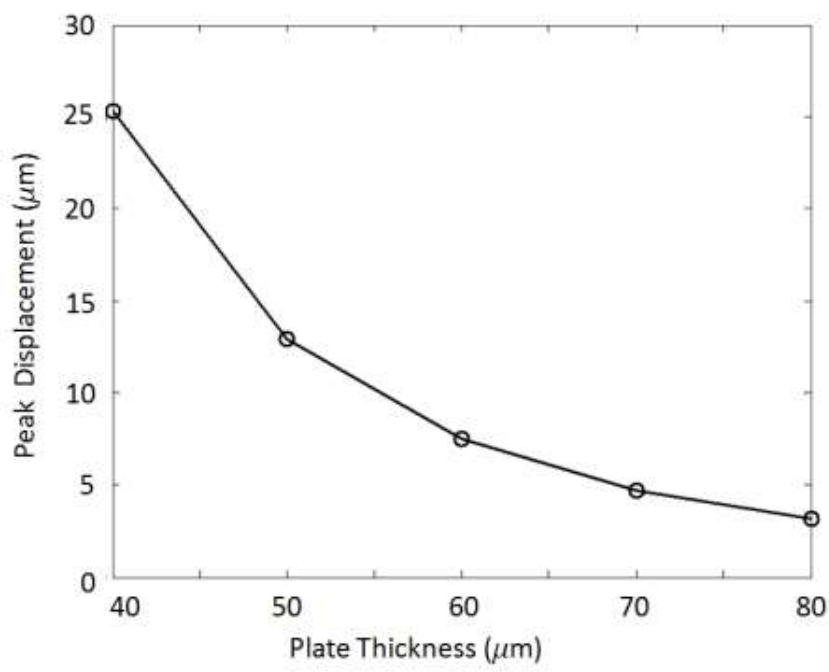

Fig. 4: Plate peak displacement

Next, we halved the CMUT plate radius into $1.0 \mathrm{~mm}$ so that the distance between the neighbouring CMUTs falls below the average two-point discrimination threshold $3 \mathrm{~mm}$, and conducted the same calculations with plate thickness altered from $80 \mu \mathrm{m}$ to $20 \mu \mathrm{m}$. Calculated spatially averaged pressure and peak displacement values are shown in Figs. 5 and 6 respectively.

$300 \mathrm{~Pa}$ is reached at the plate thickness of $28 \mu \mathrm{m}$ with the peak displacement of $6 \mu \mathrm{m}$. Though these amount of peak displacement is yet unexplored experimentarily, it seems to be in the reach of current technology. It can be said that CMUT array makes one promising candidate for tactile display device. 


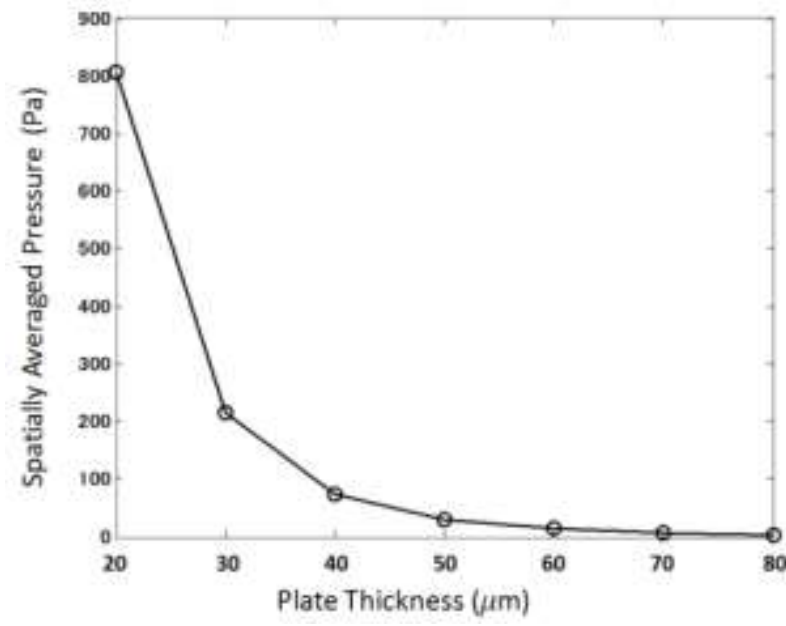

Fig. 5: Spatially averaged near-field acoustic radiation pressure when $\mathrm{r}_{0}=1.0 \mathrm{~mm}$

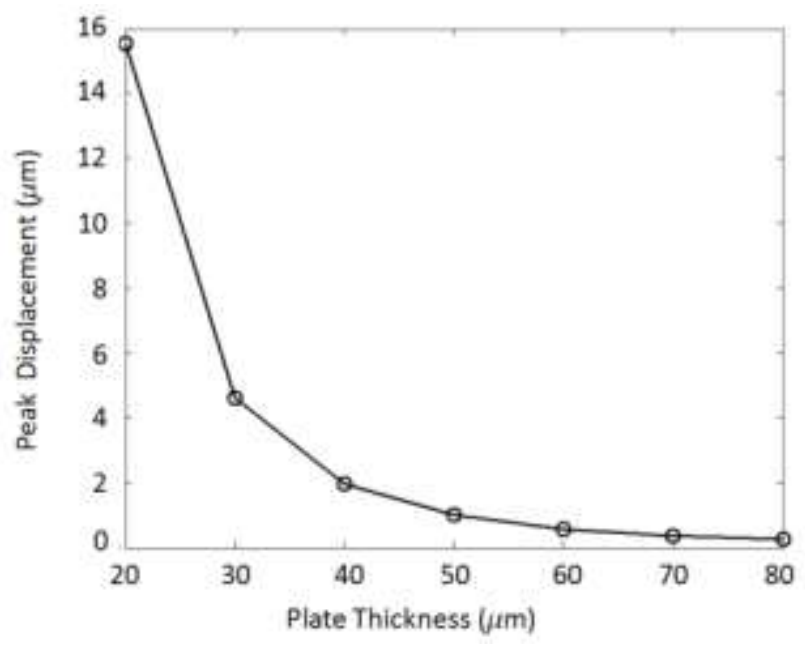

Fig. 6: Plate peak displacement when $r_{0}=1.0 \mathrm{~mm}$

\section{CONCLUSION AND FUTURE WORK}

We proposed the use of CMUT array as tactile display device and theoretically estimated the near-field acoustic radiation pressure near their surface. Results show that if we could set the average distance between the CMUT and finger surface appropriately, it is able to achieve necessary pressure for tactile sensation with spatial resolution close to or even below the average two-point discrimination threshold of the human tactile sense.

Since our fluid model is a simplified approximation which does not include detailed mechanisms such as edge pressure drop effect, etc., numerical simulation would enable more realistic estimation in the future.

\section{ACKNOWLEDGMENT}

Authors wish to thank Mr. Satoshi Nakamura for giving them the opportunity to engage with the study of near-field acoustic radiation pressure phenomena. N. K. wishes to thank Professor Dr. Sadataka Furui of Teikyo University for giving him the mention of the importance of CMUTs and to Ms. Urara Yokose of Kao Corporation who intrigued his interest to skin science.

\section{REFERENCES}

[1] Y. Hashimoto, Y. Koike and S. Ueha, "Acoustic levitation of planar objects using a longitudinal vibration mode", Journal of the Acoustical Society of Japan (E) vol. 16, no. 3, pp. 189-192, 1995. http://dx.doi.org/10.1250/ast.16.189

[2] S. Nakamura, T. Furusawa, Y. Sasao, K. Kogure and N. Kondo, "Optical measurement of acoustic radiation pressure of the near-field acoustic levitation through transparent object", arXiv:1305.6368 [physics.ins-det], 2013.

[3] Y. Shiokawa, A. Tazo, M. Konyo and T. Maeno, "Hybrid display of realistic tactile sense using ultrasonic vibrator and force display", Journal of the Japan Society of Mechanical Engineers (C), vol. 75, no. 749, pp. 132-140 (in Japanese), 2009.

[4] T. Hoshi, T. Iwamoto and H. Shinoda, "Producing tactile sensation with airborne ultra transducer array", Proceedings of the 2009 JSME Conference on Robotics and Mechatronics, 1A1-A14, 2009.

[5] A. Yilmaz, "Airborne CMUT cell design", Ph. D Thesis, Dept. Elec. \& Electron. Eng., Bilkent University, Turkey, 2014.

[6] J. Li, W. Cao, P. Liu and H. Ding, "Influence of gas inertia and edge effect on squeeze film in near field acoustic levitation", Applied Physics Letters, vol. $96,243507,2010$. http://dx.doi.org/10.1063/1.3455896

[7] I. Wygant, M. Kupnik and B. T. Khuri-Yakub, "Analytically calculating membrane displacement and the equivalent circuit model of a circular CMUT cell", IEEE International Ultrasonics Symposium Proceedings, pp. 2111-2114, 2008 http://dx.doi.org/10.1109/ultsym.2008.0522

[8] S. Timoshenko and S. Woinowsky-Kreiger, Theory of Plates and Shells, 2nd ed., New York: McGraw-Hill Higher Education, 1964.

[9] M. I. Haller and B. T. Khuri-Yakub, "A surface micromachined electrostatic ultrasonic air transducer" , Proc. IEEE Ultrason. Symp., pp. 1241-1244, 1994.

http://dx.doi.org/10.1109/ultsym.1994.401810

[10] S. Weinstein, "Intensitve and extensive aspects of tactile sensitivity as a function of body part, sex, and laterality", The Skin Senses, Kenshalo DR ed., Charles C Thomas, pp. 223-261, 1968. 